\title{
LHCb VELO Upgrade
}

\author{
Karol Hennessy ${ }^{1}$ \\ University of Liverpool, Liverpool, UK.
}

\begin{abstract}
The upgrade of the LHCb experiment, scheduled for LHC Run-III, scheduled to start in 2021, will transform the experiment to a trigger-less system reading out the full detector at $40 \mathrm{MHz}$ event rate. All data reduction algorithms will be executed in a high-level software farm enabling the detector to run at luminosities of $2 \times 10^{33} \mathrm{~cm}^{-2} \mathrm{~s}^{-1}$.

The Vertex Locator (VELO) is the silicon vertex detector surrounding the interaction region. The current detector will be replaced with a hybrid pixel system equipped with electronics capable of reading out at $40 \mathrm{MHz}$. The upgraded VELO will provide fast pattern recognition and track reconstruction to the software trigger. The silicon pixel sensors have $55 \times 55 \mu \mathrm{m}^{2}$ pitch, and are read out by the VeloPix ASIC, from the Timepix/Medipix family. The hottest region will have pixel hit rates of $900 \mathrm{Mhits} / \mathrm{s}$ yielding a total data rate of more than $3 \mathrm{Tbit} / \mathrm{s}$ for the upgraded VELO. The detector modules are located in a separate vacuum, separated from the beam vacuum by a thin custom made foil. The foil will be manufactured through milling and possibly thinned further by chemical etching.

The material budget will be minimised by the use of evaporative $\mathrm{CO}_{2}$ coolant circulating in microchannels within $400 \mu \mathrm{m}$ thick silicon substrates. The current status of the VELO upgrade is described and latest results from operation of irradiated sensor
\end{abstract} assemblies are presented.

Keywords: LHCb, VELO, Silicon Detector, Pixel, VeloPix, Microchannels, Radiation hard

\section{LHCb and its Upgrade}

The Large Hadron Collider Beauty detector [1] (figure 1] is a flavour physics detector, designed to detect decays of $b$ - and $c$-hadrons for the study of CP violation and rare decays. In pp collisions at LHC [2] energies, b $\bar{b}$ production is primarily in the forward/backward direction. LHCb has been designed as a forward arm spectrometer, to exploit this fact. LHCb is a precision experiment operating at a instantaneous luminosity of $\mathcal{L}=4 \times 10^{32} \mathrm{~cm}^{-2} \mathrm{~s}^{-1}$, and is expected to continue in its current configuration until 2018.

At the end of Run-II, many of the LHCb measurements will remain statistically dominated. Luminosity will be increased by a factor of five to $2 \times 10^{33} \mathrm{~cm}^{-2} \mathrm{~s}^{-1}$ in Run-III. The increased luminosity at $\mathrm{LHCb}$ will boosts statistics primarily in semileptonic channels containing at least one muon. However, the trigger yield for purely hadronic decay channels saturates due to energy cuts in the hardware trigger. Removing the hardware trigger eliminates the $1 \mathrm{MHz}$ bottleneck and improves the efficiency due to having full information available for the first level trigger decision Running the detector at $40 \mathrm{MHz}$ requires radical changes to many of the subdetectors of LHCb.

\section{The Current VELO detector}

The VELO [4][5] is a silicon strip detector surrounding the interaction point at $\mathrm{LHCb}$. The sensors are made of $300 \mu \mathrm{m}$

\footnotetext{
${ }^{1}$ on behalf of the LHCb VELO Upgrade collaboration
}

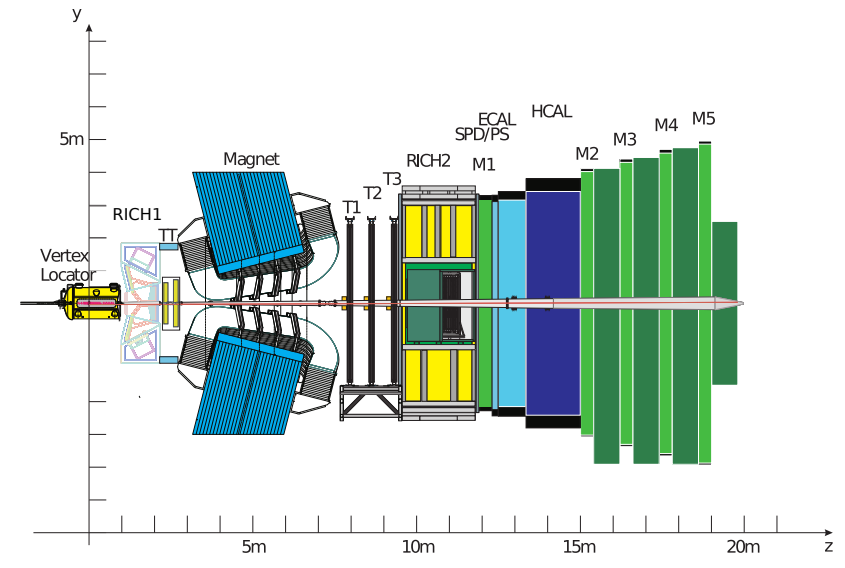

Figure 1: The LHCb detector - starting at the interaction point and proceeding downstream, we have the Vertex Locator, RICH1, TT, Magnet, T1-T2-T3 tracking stations, RICH2, Calorimeters and finally Muon chambers.

(C) Copyright CERN: reproduced with permission

$n^{+}$-on- $n$ silicon, and are positioned only $7 \mathrm{~mm}$ from the beam line during data taking. The VELO is split into two halves such that each can be retracted during LHC injection. The two VELO halves are operated in vacuum and separated from the primary LHC vacuum by means of a $300 \mu \mathrm{m}$ thick aluminium foil. The VELO is required to have excellent impact parameter resolution, essential for the reconstruction of heavy hadron decays.

The VELO consists of 42 modules placed along the beam di- 

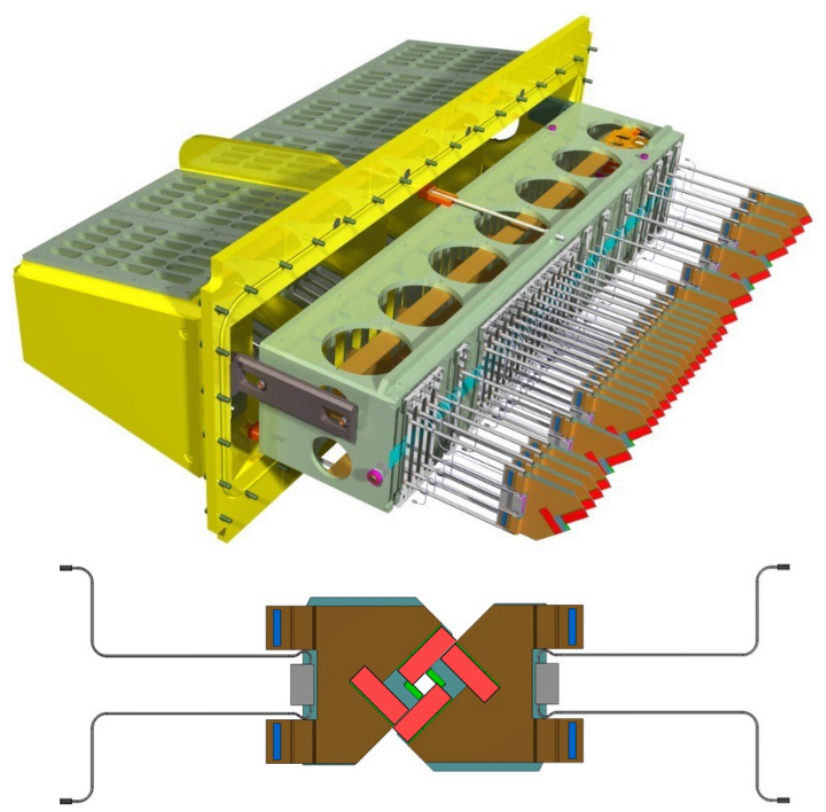

Figure 2: (top) Schematic of one half of the VELO upgrade detector. Twentysix modules are aligned along the beam direction. (bottom) Two modules in the closed position. The modules are in different z-positions along the beam line to minmise gaps in the acceptance and there is a slight overlap between the two halves which aids detector alignment.

(C) Copyright NIKHEF: reproduced with permission

rection, with the full length of the detector being approximately $1 \mathrm{~m}$. A module is made of two half-disc sensors with R-and $\Phi$-measuring geometry.

\section{VELO Upgrade}

The Vertex Locator upgrade [3] is a significant redesign from the original detector. The major changes in new detector and its predecessor are as follows:

- The detector will change from a silicon strip detector to a pixel detector.

- The detector will be closer to the beam in its closed position at $5.1 \mathrm{~mm}$ from $8.2 \mathrm{~mm}$

- The upgraded VELO will use a new VeloPix ASIC [6] that can be read out at $40 \mathrm{MHz}$ (up from $1.1 \mathrm{MHz}$ ) and a bandwidth of up to $20.4 \mathrm{~Gb} / \mathrm{s}$.

- The current detector modules are cooled with $\mathrm{CO}_{2}$ passing through a series of cooling blocks attached to the base of the module substrate. The upgraded modules will also use $\mathrm{CO}_{2}$ as a coolant, but it will pass through microchannels in a silicon substrate, directly beneath the major heat sources (VeloPix ASICs and other chips).

The upgraded VELO (shown in figure 2) will have more robust track reconstruction performance compared to a strip detector and an overall improved resolution (figure 3. This improved performance is achieved by (i) lowering the material

\begin{tabular}{lcc}
\hline Feature & Current VELO & Upgraded VELO \\
\hline Sensors & $\mathrm{R} \& \phi$ strips & Pixels \\
\# of modules & 42 & 52 \\
Detector Active area & $0.22 \mathrm{~m}^{2}$ & $0.12 \mathrm{~m}^{2}$ \\
& $\sim 172 \mathrm{k}$ strips & $\sim 41 \mathrm{M}$ pixels \\
Technology & electron collecting & electron collecting \\
& $300 \mu \mathrm{m}$ thick & $200 \mu \mathrm{m}$ thick \\
Max fluence & $3.9 \times 10^{14}$ & $8 \times 10^{15}$ \\
& $\mathrm{MeV} \cdot \mathrm{n}_{\mathrm{eq}} / \mathrm{cm}^{-2}$ & $\mathrm{MeV} \cdot \mathrm{n}_{\mathrm{eq}} / \mathrm{cm}^{-2}$ \\
HV tolerance & $500 \mathrm{~V}$ & $1000 \mathrm{~V}$ \\
ASIC Readout rate & $1 \mathrm{MHz}$ & $40 \mathrm{MHz}$ \\
Total data rate & $\sim 150 \mathrm{~Gb} / \mathrm{s}$ & $1.2 \mathrm{~Tb} / \mathrm{s}$ \\
Total Power consumption & $\sim 1 \mathrm{~kW}$ & $2.2-2.3 \mathrm{~kW}$ \\
\hline
\end{tabular}

Table 1: An outline of the major differences between the current VELO and its upgrade

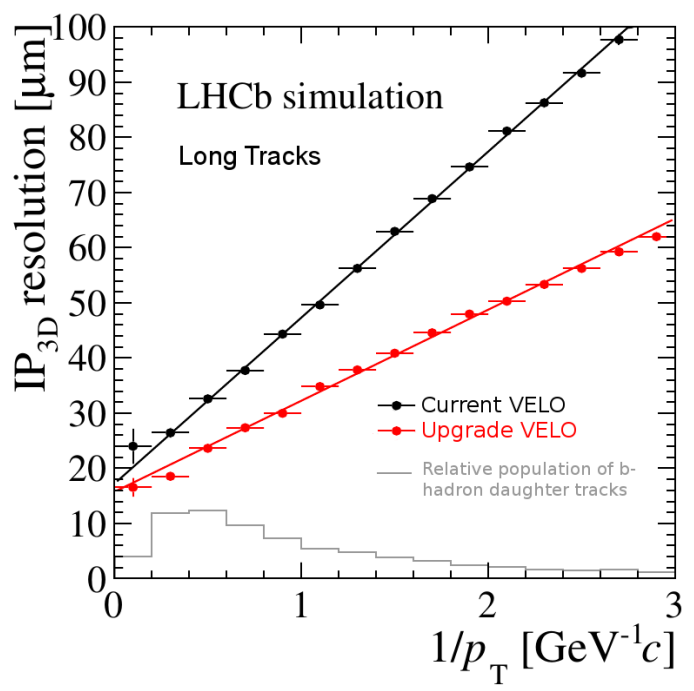

Figure 3: 3D Impact Parameter resolution vs particle inverse transverse momentum. Made using LHCb software simulation. A significant resolution improvement of the upgraded detector (red) can be seen over the current VELO (black). The upgrade operates closer to the beam line and has a lower material budget - two major contributions to the improved resolution.

(C) Copyright CERN: reproduced with permission

budget with thinner sensors and and thinner aluminium foil housing each detector half and (ii) placing the sensors closer to the beam. The closer placement of the detector modules to the beam and the higher luminosity for LHC Run III comes with some costs - the detector must be able to handle higher radiation doses; there is a higher hit occupancy due to increased particle flux. This, in turn, results in higher data rates from the detector and greater power consumption in the front-end ASICs (however, the dominant increase in data rate is due to the removal of the L0 hardware trigger). Table 1 lists some of the major differences between the current VELO detector and the upgrade.

\subsection{Modules}

A mechanical module concept is shown in figure 4 . The current design of the module consists of a carbon-fibre structure supporting a silicon microchannel substrate. Stress-relieved $\mathrm{CO}_{2}$ cooling pipes route the $\mathrm{CO}_{2}$ to and from the cooling con- 


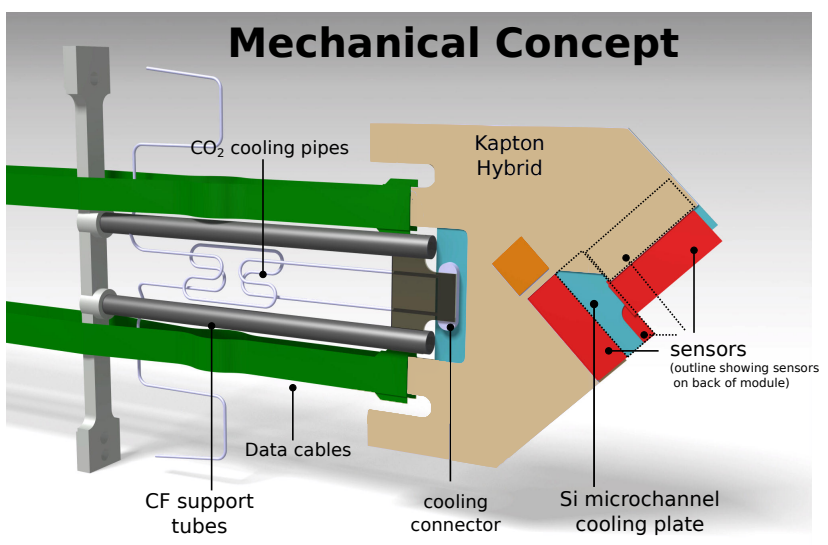

Figure 4: A conceptual 3D model of the mechanical design of a VELO upgrade module (subject to change).

(c) Copyright NIKHEF: reproduced with permission

nector which is soldered to the Si substrate. The module has four silicon sensors, read out by, and bump-bonded to twelve VeloPix ASICs. The ASICs are glued directly onto the microchannel substrate. Discrete electronics, including a GBTX chip [7] for slow control, along with power, bias, and readout circuitry will be arranged on a Kapton hybrid. When the VELO is closed, the sensors of opposing detector halves form a diamond shape with the beam passing through the centre.

\subsection{Cooling}

The primary sources of heat on the module are due to the VeloPix chips. It is estimated that the full chip will consume $<1.1 \mathrm{~W} / \mathrm{cm}^{2}$. The sensors must be kept at $-20^{\circ} \mathrm{C}$ in order to minimise the chance of thermal runaway due to radiation damage. The modules are cooled using evaporative $\mathrm{CO}_{2}$ at $-30^{\circ} \mathrm{C}$. The $\mathrm{CO}_{2}$ is passed from an inlet pipe through a cooling connector soldered to the silicon substrate. The inlet fans out to a series of parallel microchannels in the substrate which pass directly under the VeloPix chips. Near the inlet, the microchannels form a restriction region of higher pressure. This is followed by a transition region where the channels widen from $60 \mu \mathrm{m} \times 60 \mu \mathrm{m}$ to $120 \mu \mathrm{m} \times 200 \mu \mathrm{m}$ stimulating boiling of the $\mathrm{CO}_{2}$. The microchannel layout has been optimised to route the coolant directly to the site of the heat sources (the GBTX chip and VeloPix ASICs) and minimise the temperature gradient across the module. Several prototype substrates have been studied to evaluate their heat-load and pressure performance. The design was optimised to meet specifications in terms of structural integrity and cooling performance (expected maximum cooling power $>36 \mathrm{~W}$ ).

\subsection{RF Foil}

The aluminium foil serves to separate the secondary VELO vacuum from the primary LHC vacuum. The RF foil thickness has a significant impact on the impact parameter resolution. For the current detector, with a $300 \mu \mathrm{m}$ foil, particles cross an average of $\sim 3 \mathrm{~mm}$ of aluminium before the first hit in the silicon sensor. The proposed design for the upgrade VELO foil will be

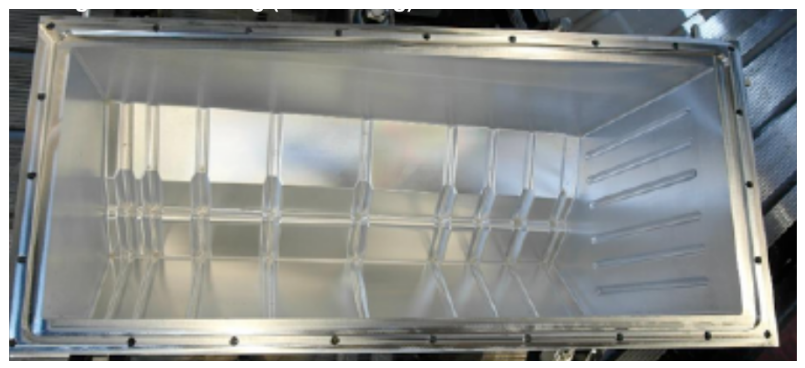

Figure 5: Half box prototype after milling. The box starts as a $180 \mathrm{~kg}$ block of aluminium and ends as a $3 \mathrm{~kg}$ piece.

(C) Copyright NIKHEF: reproduced with permission

$200 \mu \mathrm{m}$ thick. Studies on the foil manufacture have been done - including milling the foil from a solid block of aluminium (a prototype half-box is show in figure 5, and chemically etching areas of the foil which have greatest impact on the IP resolution.

\section{Beam tests}

A campaign of beam tests were performed throughout 2014 and 2015 to characterise the performance of candidate VELO upgrade sensors. The two major vendors studied were Hamamatsu (HPK) and Micron. Testing was performed with a Timepix 3 telescope. Timepix 3 is a precursor of the VeloPix ASIC but runs at a lower data rate $(80 \mathrm{Mhit} / \mathrm{s}$, compared to 900 Mhits/s for VeloPix). VeloPix has binary readout, whereas Timepix 3 has analogue readout yielding a better time and spatial resolution. Timepix 3 has the same pixel geometry as VeloPix and therefore was an ideal test-bed for characterising the VELO upgrade sensors ${ }^{2}$.

The upgrade sensors will receive an highly non-uniform radiation dose up to $8 \times 10^{15} \mathrm{MeV} \cdot \mathrm{n}_{\mathrm{eq}} / \mathrm{cm}^{-2}$ during their lifetime. At this dose, the sensors are expected to retain a $99 \%$ hit efficiency at up to $1000 \mathrm{~V}$ bias voltage without suffering breakdown. The HPK and Micron sensors tested with the Timepix3 telescope were compared un-irradiated and irradiated to their maximum dose $\left(8 \times 10^{15} \mathrm{MeV} \cdot \mathrm{n}_{\mathrm{eq}} / \mathrm{cm}^{-2}\right.$ equivalent to an integrated luminosity of $50 \mathrm{fb}^{-1}$ ). The single hit resolution of detectors is shown in figure 6 and the charge collection performance is shown in figure 7. Sensor tests defined a baseline choice of $200 \mu \mathrm{m}$ n-on-p silicon as suitable for the VELO upgrade. This is subject to change if, for example, a similarly performing thinner sensor be found.

\section{VeloPix and DAQ}

At the heart of new VELO upgrade electronic design is the new VeloPix ASIC [6]. The VeloPix is based on the Timepix3 ASIC and has a data-driven readout. The $256 \times 256$ pixel array is arranged into groups of $2 \times 4$ pixels called SuperPixels. Binary hit information is time-stamped, addressed and read out as SuperPixels packets. Arranging the data in this way reduces

\footnotetext{
${ }^{2}$ The VeloPix design had not been finalised at the time of sensor testing
} 


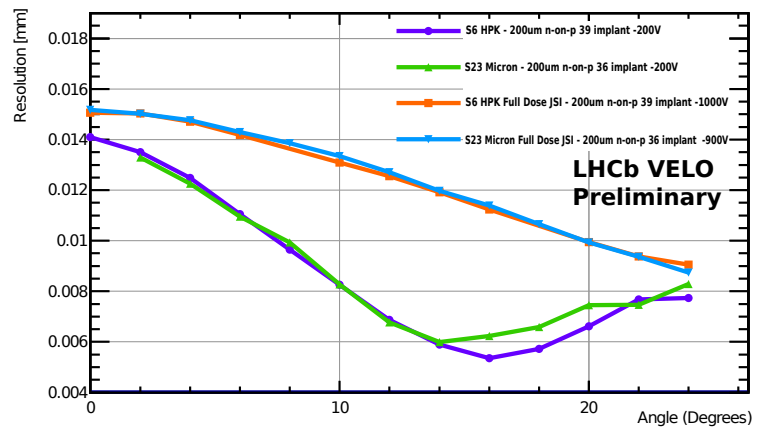

Figure 6: Single hit resolution of two test sensors before (Micron - green, HPK - purple) and after (Micron - blue, HPK - orange) irradiation as a function of incident beam angle. Both sensors show very similar performance. An approximate factor of two degradation in resolution can be seen in the worst case. The sensors were irradiated to $8 \times 10^{15} \mathrm{MeV} \cdot \mathrm{n}_{\mathrm{eq}} / \mathrm{cm}^{-2}$.

(c) Copyright CERN: reproduced with permission

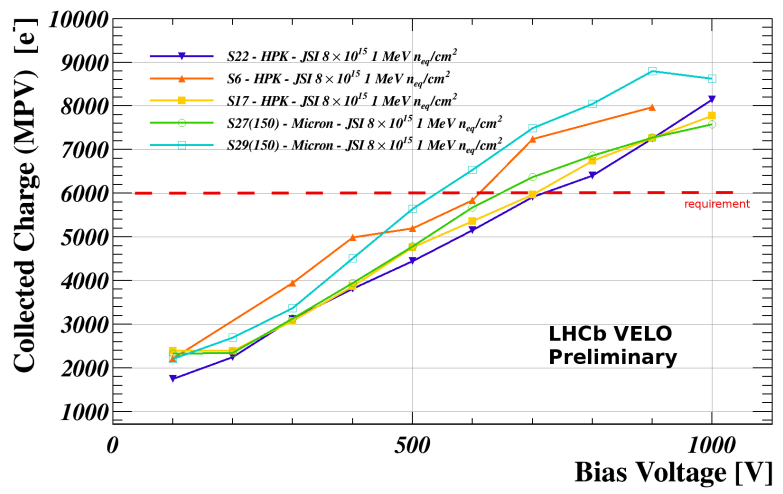

Figure 7: Collected charge (determined from Most Probable Value of their Landau distributions) as a function of Bias voltage after irradiation at full dose $\left(8 \times 10^{15} \mathrm{MeV} \cdot \mathrm{n}_{\mathrm{eq}} / \mathrm{cm}^{-2}\right)$. Three Hamamatsu $(200 \mu \mathrm{m})$ and two Micron $(150 \mu \mathrm{m})$ sensors were tested. All sensors pass the 6000 electron requirement (represented by the dashed line) at a voltage limit of $1000 \mathrm{~V}$.

(c) Copyright CERN: reproduced with permission

the output bandwidth by $\approx 30 \%$ over standard pixel readout. The data readout latency varies as a function of SuperPixel hit position, and hits closer to the beam take longer to reach the end-of-column readout logic. In addition, the hits will be disordered in time.

The VeloPix will be read out using a PCIe40 readout board [8] which has been designed as a generic readout board for the LHCb upgrade. The PCIe40 firmware is designed as a series of common components with the option for user-specific data processing. The common components deal with accepting the input data from the detector over the GBT protocol [9], errorchecking, dealing with reset signals, and preparing the data for the event builder and computing farm. The user-specific code would, for example, perform zero-suppression or similar datareduction techniques.

For the VELO upgrade, some of the common input blocks have had to be replaced as the GBT protocol is not used for reasons of power consumption at the front-end. A simpler protocol
- Gigabit Wireline Transmitter (GWT) has been used instead. The VELO user-specific blocks include primarily (i) data descrambling, decoding and parity checking, (ii) a time-ordering router which re-sequences the data coming from the continuous readout of the VeloPix chip, and (iii) a hit isolation tagger which performs a simplified type of clusterisation to reduce the load downstream in the reconstruction software running on the CPU farm. Resource utilisation within the FPGA is within acceptable tolerances and studies of packet loss rates are ongoing. Preparations are being made to assess VeloPix readiness.

\section{Conclusion}

The VELO upgrade detector makes significant improvements on its predecessor. Improved tracking performance is achieved using a pixel sensor closer to the interaction region $(5.1 \mathrm{~mm})$ but with a reduced material budget. This leads to an even higher and non-uniform radiation tolerance requirement. As such the sensors and readout chips are cooled using a $\mathrm{CO}_{2}$ coolant via a microchannel silicon substrate. Candidate sensors meeting the required performance constraints have been identified. Significant progress has been made to both control and read out the forthcoming VeloPix chip which will be tested in the coming months.

\section{Acknowledgements}

Thanks to Science Technology Funding Council (STFC) of the UK, University of Liverpool, CERN and associated institutes of $\mathrm{LHCb}$.

\section{References}

[1] The LHCb Collaboration, The LHCb Detector at the LHC, Published in Journal of Instrumentation (INST), 2008 JINST 3 S08005

[2] CERN, The Cern Large Hadron Collider: Accelerator and Experiments, 2008, JINST 3 S08001

[3] The LHCb Collaboration, LHCb VELO Upgrade Technical Design Report, CERN-LHCC-2013-021 (2013)

[4] LHCb VELO Collaboration, LHCb VELO Technical Design Report, CERN/LHCC 2001-011 (2001)

[5] K. Carvalho Akiba, LHCb VELO: Radiation Damage Effects and Operations in LHC Run 2, these proceedings

[6] T. Poikela, M. De Gaspari, J. Plosila, T. Westerlund, R. Ballabriga, J. Buytaert, M. Campbell, X. Llopart, K. Wyllie, V. Gromov, M. van Beuzekom and V. Zivkovic, VeloPix: the pixel ASIC for the LHCb upgrade, Journal of Instrumentation 10 (2015), no. 01, C01057

[7] P. Moreira, J. Christiansen, K. Wyllie, GBTX Manual, https://espace.cern.ch/GBT-Project/GBTX/Manuals/gbtxManual.pdf

[8] M Bellato, G Collazuol, I D'Antone, P Durante, D Galli, B Jost, I Lax, G Liu, U Marconi, N Neufeld, A PCIe Gen3 based readout for the LHCb upgrade, Journal of Physics: Conference Series, 513 (2014), no. 1, 012023

[9] P. Moreira et al, The GBT Project, Available at http://cds.cern.ch/record/1235836 